\title{
Factores biológicos en la epilepsia que provocan déficit de la inteligencia
}

\author{
Biological factors in epilepsy causing intelligence deficit
}

\author{
Salvador González-Pal ${ }^{1 *}$, Reinaldo Fabelo-Roche ${ }^{1}$, Erick González-Delgado ${ }^{2}$, Serguei Iglesias-Moré $^{3}$ \\ 1 Facultad Calixto García Iñiguez. Instituto Superior de Ciencias Médicas de La Habana. La Habana, Cuba. \\ 2 Servicio de Neurología. Hospital Hermanos Ameijeiras. La Habana, Cuba. \\ 3 Facultad Manuel Fajardo. Instituto Superior de Ciencias Médicas de La Habana. La Habana, Cuba.
}

\section{Resumen}

Mediante el estudio de un grupo de pacientes adultos con epilepsia, se investigan los niveles bajos de coeficiente de inteligencia (CI) e índice de deterioro cognitivo (ID) y su relación con un conjunto de variables biológicas. El objetivo es identificar las variables biológicas a largo plazo para disminuirlas. Utilizamos el $\mathrm{Cl}$ y el ID de Wechsler que se aplicó a 200 pacientes diagnosticados de epilepsia, divididos en dos grupos, los que tenían $\mathrm{Cl}<89$ y $>90$, y los que tenían ID positivo y los que no. Estas variables se analizaron según factores biológicos. Los datos se procesaron con diferentes estadígrafos. Del total de pacientes estudiados, 109 (54.5\%) tienen un $\mathrm{Cl}<89$ y $50 \%$ un índice de deterioro cognitivo. Los factores relacionados en los pacientes con $\mathrm{Cl}$ menor de $<89$ eran, entre otros, inicio de las crisis por debajo de los 15 años de edad, más de 15 años de evolución de las crisis, elevada frecuencia de las crisis, antecedentes de estado de mal epiléptico y las crisis parciales secundariamente generalizadas. Los enfermos con crisis focales secundariamente generalizadas tienen más deterioro que los que sufren de crisis focales o generalizadas, ANOVA $p=0.0029$ muy significativo. La mitad de los pacientes presentan $\mathrm{Cl}$ normal bajo, así como deterioro. Se considera que, aunque no es evidente el deterioro detectado mediante el WAIS, es importante conocer su existencia para controlar los factores de riesgo biológicos.

Palabras clave: coeficiente de inteligencia, epilepsia, deterioro cognitivo, factores de riesgo

\section{Abstract}

In a group of adult patients suffering from epilepsy, low intelligence quotient (IQ) levels, the cognitive deterioration rate as well as its relation to a number of biological variables are studied. The aim of this research is to determine the long term biological variables in order to reduce them. We used the IQ and Wechsler's Deterioration index (DI) to study 200 patients diagnosed with epilepsy. The patients were divided into two groups, a first group included those with $1 Q<89$ and $>90$ and the second patients with or without a positive DI. These variables were analyzed according to different biological factors. Data was processed using different statistical methods. Out of the total of studied patients, $54.5 \%$ showed an IQ $<89$ and $50 \%$ a cognitive deterioration rate. Factors related to the patients with an IQ lower than 89 were among others the following: The start of the seizures before the age of 15 , having suffered from epileptic seizures for more than 15 years, a high frequency of seizures, and personal past history of status epilepticus as well as suffering from secondarily generalized partial epileptic seizures. Patients suffering from generalized secondarily focal epileptic seizures show a higher cognitive deterioration than those suffering focal or generalized seizures, ANOVA $p=0.0029$, which is highly meaningful. Half of the patients show a normal-low IQ, as well as some cognitive deterioration. Although the deterioration detected by using WAIS was not evident, it is important to be aware of the fact that there are biological risk factors in order to be able to control them.

Keywords: intelligence quotient (IQ), epilepsy, cognitive deterioration, risk factors

\footnotetext{
* Correspondencia: sglezpal@infomed.sld.cu. Facultad Calixto García Iñíguez. Instituto Superior de Ciencias Médica de La Habana. Avenida de Presidentes y Ave Universidad. Vedado. La Habana, Cuba Recibido: 20-02-11. Revisión desde: 07-03-11. Aceptado: 20-06-11
}

DOI: $10.5839 /$ rcnp.2011.0601.05 


\section{Introducción}

En el siglo XVIII, Jean-Étienne Dominique Esquirol, psiquiatra francés, describe las "Demencias de enfermos mentales" y, entre estos, incluye a los pacientes con epilepsia. El deterioro de la inteligencia, descrito en siglo XIX como demencia, era una asociación muy frecuente en los pacientes con epilepsia en este siglo. Aunque la causa de estos estados demenciales no estaba muy bien definida, se sugiere que pudo estar relacionada con dos procesos frecuentes en ese siglo: la elevada prevalencia de la neurosífilis y el internamiento de los enfermos con epilepsia en los manicomios, centros que comenzaron a usarse en esa época. El tema del deterioro en la epilepsia ha sido tratado a lo largo de los años por psiquiatras y neurólogos, pero no es hasta fines del siglo pasado y principios de este que se resume como producto de un fenómeno multifactorial, relacionado en general con factores biológicos, medicamentosos y psicosociales (Binnie, Debets, Engelsman et al., 1989; Brown, 2006; Caramelli \& P, M.C., 2005; Hermann, Sidenberg, \& Bell, 2000; Hermann \& Whitman, 1984; Hermann \& Whitman, 1991; Ure, 2004).

El concepto de deterioro de la inteligencia ha sido definido como la "caída o pérdida de habilidades intelectuales anteriormente existentes por causas patológicas" (CamposCastello, 2006), lo que excluye la pérdida de aquellas funciones relacionadas con al proceso vital de desarrollo de involución del humano (envejecimiento). En el concepto de deterioro intelectual se incluyen tanto el no desarrollo como la disminución en diversos grados de cualquiera de las múltiples funciones de la inteligencia. Mientras que se considera que las causas del deterioro son generalmente multifactoriales y su pronóstico es totalmente diferente si se trata de deterioro por un fenómeno de "interferencia", como el que puede producir una crisis eléctrica no clínica, o el "estructural", como el que ocurre en las epilepsias encefalopáticas (Brown, 2006).

Para determinar el deterioro de la Inteligencia nos valemos del Wechsler, prueba que nos permite obtener dos "índices" de deterioro: el coeficiente de inteligencia $(\mathrm{Cl})$ y el índice de deterioro. De ambos hemos hecho referencia en estudios anteriores (González-Pal, Quintana-Mendoza, Fabelo-Roche, Delgado \& Moré-Iglesias, 2009; Quintana-Mendoza, GonzálezPal \& González-Rodríguez, 2004).

El coeficiente de inteligencia total $\mathrm{Cl}$ se divide en $\mathrm{Cl}$ verbal y ejecutivo. Dentro del $\mathrm{Cl}$ verbal se incluyen: la información, la comprensión, la aritmética, las similaridades o semejanzas, y los dígitos y el vocabulario. Dentro del $\mathrm{Cl}$ ejecutivo: dígitos, símbolos, completar figuras, diseño de bloques, arreglos de figuras, armar objetos (Wechsller, 1955). En general, la disminución del $\mathrm{Cl}$ en la escala del Whechsler habla de "...alteraciones negativas en el desarrollo de las habilidades intelectuales..."

El índice de deterioro se obtiene mediante un conjunto de funciones existentes dentro de las escalas verbal y ejecutiva, que no disminuyen con la edad y se deben mantener similares durante las distintas etapas de la vida (SM): la información, la comprensión, ensamblar objetos y completar figuras. Otro grupo de funciones, incluidas también dentro de las verbales y ejecutivas, no se mantienen y disminuyen con la edad del paciente (NSM): dígito símbolo, aritmética, dígitos, diseño de bloques. Cuando la correlación entre el SM y el NSM es mayor para la segunda, indica que existe deterioro (QuintanaMendoza, et al., 2004; Wechsler, 1955). El índice de deterioro positivo en esta prueba es expresión de "[... ] caída o pérdida de habilidades intelectuales anteriormente existentes"?

\section{Objetivos}

\section{General}

Determinar el índice de deterioro y el coeficiente de inteligencia en pacientes adultos con epilepsia.

\section{Específicos}

Precisar los factores de riesgo biológico de la población estudiada relacionados con la etiología (antecedentes de parto distócico, trauma craneal, alcoholismo crónico), edad de inicio de la epilepsia por debajo de los 15 años, más de 10 años de duración de la enfermedad, tipos de crisis de epilepsia, tipos de síndromes epilépticos, frecuencia elevada de las crisis en los últimos tres años, presencia de más de 100 crisis en un año o estado de mal epiléptico.

Determinar, en los pacientes con un $\mathrm{Cl}$ por debajo de 89, cuáles son los factores de riesgo biológicos que actúan sobre estos. Relacionar en estos enfermos el índice de deterioro positivo con los factores de riesgo biológico.

\section{Material y método}

Fueron estudiados 200 pacientes con epilepsia que asistieron, de forma consecutiva, a la consulta de Epilepsia del Centro de Tratamientos Especializados de la Universidad Médica de La Habana, entre junio de 2009 y mayo de 2010. El diagnóstico de epilepsia fue realizado con los criterios de la Liga Internacional contra la Epilepsia (ILAE).

Se les aplicó a todos los pacientes el cuestionario de epilepsia para determinar edad, sexo y raza, así como factores de riesgo biológicos: etiología (antecedentes de parto distócico, trauma craneal, alcoholismo crónico), edad de inicio de la epilepsia por debajo de los 15 años, más de 10 años de duración de la enfermedad, tipos de crisis de epilepsia, tipos de síndromes epilépticos, frecuencia elevada de las crisis en los últimos tres años, presencia de más de 100 crisis en un año o estado de mal epiléptico. Los tipos de crisis y síndromes epilépticos fueron clasificados según Propuesta de Clasificación de 2001 (Acevedo, Campos, Mesa \& Nuñez, 2006; Beghi, 2009; Engel, 2006). A todos los pacientes se les aplicó la Escala de Inteligencia para adultos de Wechsler (Wechsler, 1955).

Escala de inteligencia para adultos de Wechsler: resultados del $\mathrm{Cl}$ verbal, el $\mathrm{Cl}$ ejecutivo, el $\mathrm{Cl}$ total. El Índice de inteligencia fue agrupado de la siguiente manera: grupo $1=$ Retraso mental (RM) (Cl menor de 67); grupo 2 = Fronterizo o Boderline ( $\mathrm{Cl}$ entre 68 y 80 ); grupo 3 Normal Bajo ( $\mathrm{Cl}$ entre 81 y 89 ); grupo $3=$ Normal $(\mathrm{Cl}$ entre 90 y 109$)$; grupo $4=$ Normal alto $(\mathrm{Cl}$ más de 110). Para el análisis de diferentes variables de factores de riesgo estudiadas, se reagruparon los resultados del $\mathrm{Cl}$ total en dos grandes grupos: los que tenían un $\mathrm{Cl}$ por debajo de 89 (Normal bajo y Retraso Mental) y por encima de 90 (Normal y Normal alto). La disminución del coeficiente de inteligencia en 
esta escala es índice de alteraciones en el desarrollo de ciertas habilidades intelectuales.

Índice de deterioro de Wechsler: el índice de deterioro es determinado mediante el estudio de funciones de dos tipos, las que varían respecto de la edad y no se mantienen (dígito símbolo, aritmética, dígitos, diseño de bloques), son conocidas como NSM (No Se Mantienen) y las que no varían y se mantienen (información, comprensión, ensamblar objetos y completar figuras), conocidas como SM (Se Mantienen). Estas últimas son las funciones afectadas por el deterioro. El procedimiento se basa en aplicar la fórmula (SM - NSM)/SM X100. Este resultado se considera que si es menor de $10 \%$ es normal, entre 10 y $20 \%$ posible deterioro y más de 21 deterioro evidente. Hay que considerar que con la edad aparecen valores calculados que deben de ser descontados (menos $20-24=0.5 ; 25-$ $29=0 ; 30-34=4 ; 35-39=4 ; 40-44=9 ; 50-49=14$ y $55-59$ =16). Para procesamiento y análisis de los datos se agruparon los pacientes con posible deterioro y definitivo en un grupo, mientras que los que no tenían ningún tipo de deterioro formaron el grupo control.

Se determinaron las siguientes variables biológicas: lesiones causales de epilepsia (alcoholismo crónico, lesiones por parto distócico, traumas craneales severos o moderados y encefalitis), edad de inicio de las crisis (menos de 15 años y más de 15 años), tiempo de evolución de las crisis (menos de 15 años y más de 15 años) frecuencia elevada de crisis (más de tres crisis por mes) en los últimos tres años, tipos de crisis de epilepsia y síndromes epilépticos.

\section{Procesamiento estadístico}

Se comparó cada variable biológica, psicosocial y medicamentosa de forma individual con los que tenían un $\mathrm{Cl}$ por encima de 90 y con los que lo tenían por debajo de 89. Además, se comparó con los que tenían deterioro y los que no lo tenían. Los datos se procesaron mediante análisis estadístico univariado y multivariado con el cálculo de distribuciones de frecuencia y porcentajes en las variables cualitativas, y medidas de tendencia central (media aritmética) y de dispersión (desviación estándar) en las variables cuantitativas. Las comparaciones entre dos variables se realizaron mediante análisis bivariado con prueba de t para medias aritméticas Fisher y prueba Chi-cuadrado para variables cualitativas. Para determinar asociación o relación entre variables se utilizó la prueba de Chi-cuadrado con corrección de Yates. Si existía asociación, se determinó la presencia y cuantificación de riesgo significativo mediante la prueba de productos cruzados u Odds Ratio, en la que se utilizó aproximación de Woolf. Se aceptó un nivel de significación del 5\% y, en el caso del Odds Ratio, un intervalo de confianza de 95\%, calculado por el método de Woolf.

\section{Resultados}

Edad: la edad promedio de los 200 pacientes es de 32,57 . Con menos de 25 años, 67 pacientes (33,5\%). Entre 26 y 35, 55 pacientes $(27,5 \%)$. Entre 36 y 45 años, 53 pacientes $(26,5 \%)$. Con más de 46 años, 25 pacientes $(12,5 \%)$.

Sexo: del total, 82 (41\%) son del sexo femenino y 118 (59\%) del masculino.
Grupo étnico: predomina la raza blanca con 138 pacientes (69\%), la mestiza $49(24,5 \%)$ y la negra $13(6,5 \%)$.

Coeficiente de inteligencia. De los 200 pacientes estudiados, 22 (11\%) pacientes sufren de retraso mental ligero con $\mathrm{Cl}$ menor de 67; en $44(22 \%)$ se encuentra $\mathrm{Cl}$ entre 68 y 80 considerados Fronterizo o Boderline; 44 (22\%) tienen $\mathrm{Cl}$ entre 81 y 89 , considerado Normal Bajo; 83 (41,5\%) tienen Ci entre 90 y 109, considerado Normal, y $7(3,5 \%)$ tienen $\mathrm{Cl}$ por encima de 110 , considerado Normal Alto (ver gráfico 1).

Índice de deterioro: sin deterioro 100 pacientes (50\%), con posible deterioro 44 pacientes $(44 \%)$ y con deterioro evidente 56 pacientes (56\%), ambos grupos incluyen a 100 pacientes con signos de deterioro (50\%) (ver gráfico 2).

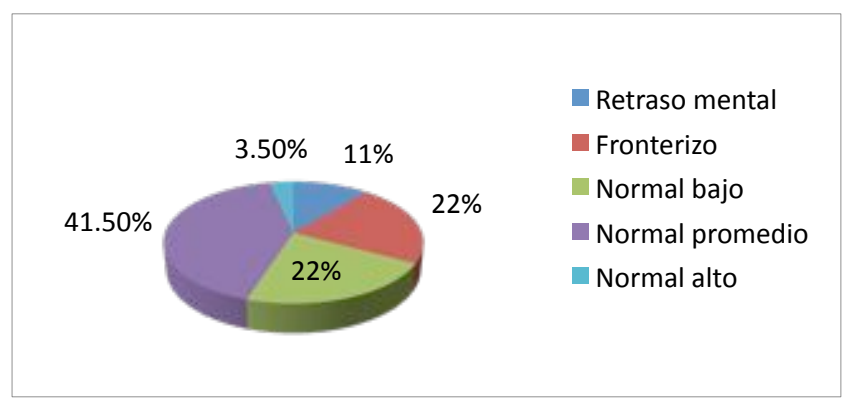

Gráfico 1. Coeficiente de Inteligencia

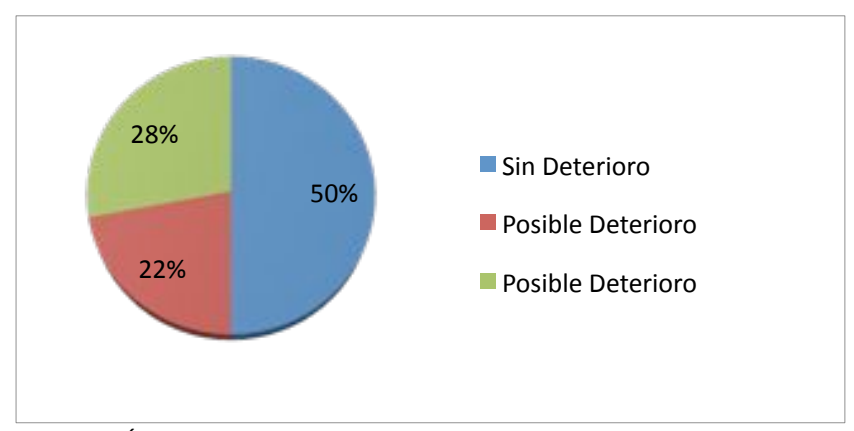

Gráfico 2. Índice de deterioro.

A partir de este momento se analiza la frecuencia de presentación de los factores de riesgo predefinidos en lo siguientes cuatro grupos de pacientes:

Grupo 1. Coeficiente de inteligencia por debajo de 89.

Grupo 2. Coeficiente de inteligencia por encima de 90.

Grupo 3. Pacientes sin deterioro de la inteligencia.

Grupo 4. Pacientes con deterioro de la inteligencia.

\section{Variables biológicas}

\section{Noxas cerebrales}

\section{Antecedentes de parto distócico con anoxia perinatal}

Coeficiente de inteligencia total. De los 200 pacientes con epilepsia, 38 (19\%) tenían antecedentes de distocia del parto; de estos, 22 (57,9\%) con un $\mathrm{Cl}$ por debajo de 89 normal bajo, limítrofe y con retraso mental ligero, y $16(42,1 \%)$ con $\mathrm{Cl}$ por encima de 90 (Normal y Normal alto). De los 162 (81\%) que no tenían antecedentes de distocia al nacer, $87(53,7 \%)$ tenían un 
$\mathrm{Cl}$ por debajo de 89 y $75(46,3 \%)$ un $\mathrm{Cl}$ por encima de 90 . La comparación de ambos grupos mostró al chi2 $p=0.0 .7749$ no significativo.

Índice de deterioro. De los 38 (19\%) pacientes con distocia en el parto, $17(44,7 \%)$ tenían signos de deterioro y 21 $(55,3 \%)$ no los presentaban. De los 162 pacientes sin antecedentes de lesión perinatal, 83 (51,2\%) tenían deterioro y 79 $(48,8 \%)$ no tenía signos de deterioro. La comparación de ambos grupos mediante Chi2 mostró $\mathrm{p}=0.5887$, no significativa.

Ninguno de los $\mathrm{Cl}$ ni el índice de deterioro se vieron afectados con el factor de riesgo de parto distócico.

\section{Antecedentes de trauma craneoencefálico que pudo provocar lesión cerebral y epilepsia}

Coeficiente de inteligencia ( $\mathrm{Cl}$ ). Del total de pacientes estudiados, 19 (9,5\%) refirieron antecedentes de trauma craneal severo y moderado; de éstos, 11 (57,9\%) tenían un $\mathrm{Cl}$ por debajo de 89 , y $8(42,1 \%)$ por encima de 90 . De los restantes, 181 (90,5\%) sin antecedentes de trauma craneal, $98(54,1 \%)$ tenían $\mathrm{Cl}$ por debajo de 89 , y $83(45,9 \%)$ por encima de 90 . Ambos grupos fueron comparados y se les aplicó Fischer con $\mathrm{P}=0.8126$, no significativa.

Índice de deterioro. Total de pacientes con trauma cráneo encefálico severo y moderado 19 (9,5\%), de los cuales 9 $(47,4 \%)$ tienen deterioro y $10(52,6 \%)$ no lo tienen. De los 181 $(90 ; 5 \%)$ que no refieren trauma craneoencefálico severo, 91 $(50,3 \%)$ tienen deterioro y $90(49,7 \%)$ no lo tienen. La comparación de ambos grupos mediante Chi2 mostró $p=0.8094$, no significativa.

Antecedentes de encefalitis bacteriana que pudo provocar lesión cerebral y epilepsia

Coeficiente de inteligencia ( $\mathrm{Cl}$ ). De los 200 pacientes estudiados, solo $21(10,5 \%)$ tenían antecedentes de encefalitis; de éstos, 12 $(57,1 \%)$ tenían un $\mathrm{Cl}$ por debajo de 89 , y $9(42,9 \%)$ por encima de 90; de los restantes $179(89,5 \%)$ pacientes, 97 (54,2\%) tenían un $\mathrm{Cl}$ por debajo de 89 y $82(45,8 \%)$ un $\mathrm{Cl}$ por encima de 90 . La comparación entre los grupos mostró Chi2 $p=0.9797$, no significativa.

Índice de deterioro. De los 21 (10,5\%) pacientes con antecedentes de encefalitis, $12(57,1 \%)$ tenían índice de deterioro y $9(42,9 \%)$ no lo tenían. De los 179 sin antecedentes de encefalitis, $88(49,2 \%)$ tenían deterioro y $91(50,8 \%)$ no lo tenían. Chi2 $\mathrm{p}=0.6446$ considerada no significativa.

\section{Antecedentes de alcoholismo crónico que pudo provocar lesión cerebral y epilepsia}

Coeficiente de inteligencia ( $\mathrm{Cl}$ ). De los 200 pacientes estudiados, 21 (10.5\%) tenían antecedentes de alcoholismo crónico, 16 $(76,2 \%)$ tenían $\mathrm{Cl}$ por debajo de 89 y solo $5(23,8 \%)$ por encima de 90 . De los restantes 179 pacientes $(89,5 \%)$ que no tenían alcoholismo crónico, 93 (51,9\%) tenían $\mathrm{Cl}$ por debajo de 89 , mientras que $86(48,1 \%)$ tenían $\mathrm{Cl}$ mayor de 90 . La comparación mediante Chi2 entre los grupos con y sin antecedentes de alcoholismo mostró una $\mathrm{p}=0.0603$.

Índice de deterioro. De los 21 (10.5\%) pacientes con alcoholismo, $14(66,7 \%)$ tenían índice de deterioro positivo y sólo 7 (33,3\%) no lo tenían. De los restantes 179 (89,5\%) que no tenían alcoholismo crónico, 86 (48,1\%) presentaban deterioro, mientras que $93(51,9 \%) \%)$ no lo presentaban. La comparación entre los grupos con y sin antecedentes de alcoholismo mostró en el Chi2 $p=0.1664$, considerada no significativa.

\section{Inicio de las crisis de epilepsia por debajo de los 15 años de edad}

Coeficiente de inteligencia(CI). De 200 pacientes con epilepsia, $67(33,5 \%)$ iniciaron sus crisis de epilepsia por encima de los 15 años de edad, de éstos, 29 (43,3\%) tienen un $\mathrm{Cl}$ menor de 89, mientras que $38(56,7 \%)$ tienen uno mayor de 90 . De los 133 $(66,5 \%)$ pacientes que comenzaron sus crisis por debajo de los 15 años, 80 (60,2\%) tienen un $\mathrm{Cl}$ menor de 89, y 53 (39,8\%) un $\mathrm{Cl}$ mayor de 90 . Ambos grupos fueron comparados con Chi2 y mostró una $\mathrm{p}=0.0348$, considerada como significativa. Odds Ratio $=0.5056$ no significativo (ver gráfico 3 ). En pacientes en que la epilepsia comenzó por debajo de los 15 años predomina un coeficiente de inteligencia por debajo de 89 , mientras que en aquellos en que empezó por encima de los 15 predomina un $\mathrm{Cl}$ por encima de 90 .

Índice de deterioro. De 67 (33,5\%) pacientes que iniciaron sus crisis de epilepsia por encima de los 15 años de edad, $32(47,8 \%)$ tienen posible deterioro y deterioro definitivo, mientras que 35 (52,8\%) no lo tienen. De los 133 (66,5\%) que iniciaron sus crisis por encima de los 15 años, 68 (51,1\%) tenían deterioro y $65(48,9 \%)$ no lo tenían. Los grupos fueron comparados mediante Chi2 $p=0.73645$, considerada no significativa.

\section{Más tiempo de evolución de las crisis (más de 15 años)}

Coeficiente de inteligencia (CI). De 200 pacientes estudiados, 106 (53\%) tenían menos de 15 años de evolución de las crisis; de estos, $50(47,2 \%)$ con $\mathrm{Cl}$ menor de 89 y $56(52,8 \%)$ con $\mathrm{Cl}$ mayor de 90 . Tenían 15 y más años de evolución de las crisis 94 pacientes (47\%), de los cuales $59(62,8 \%)$ tenían un $\mathrm{Cl}$ menor de 89 , y $35(37,2 \%)$ uno mayor de 90 . Los grupos se compararon con Chi2 $\mathrm{p}=0.386$, considerado significativo. Odds Ratio = 0.5297 (ver gráfico 4).

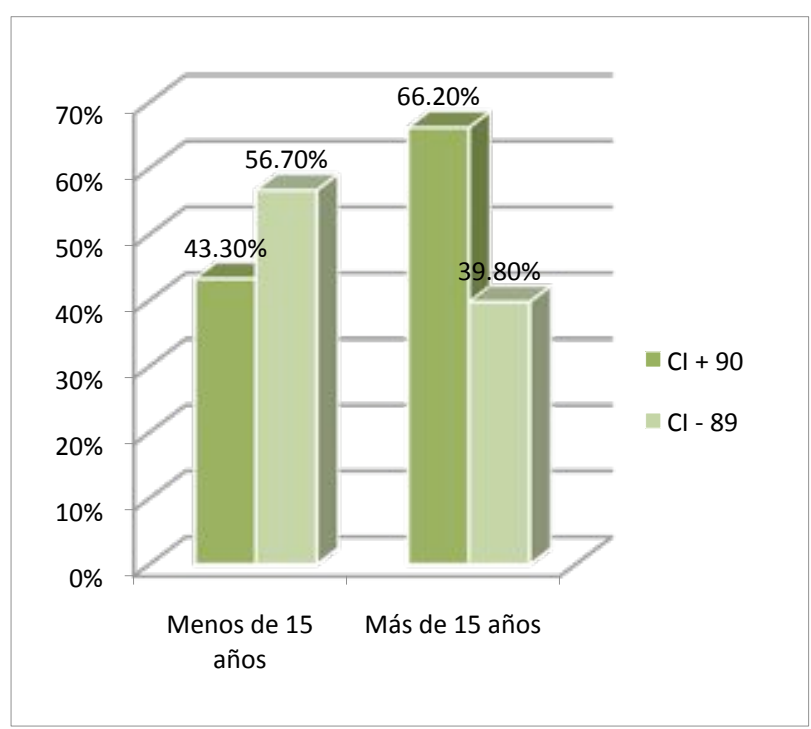

Gráfico 3. Relación del coeficiente de inteligencia del WAIS con la edad de inicio de las crisis de epilepsia. ${ }^{*} \mathrm{Chi} 2 \mathrm{p}=0.348$. 


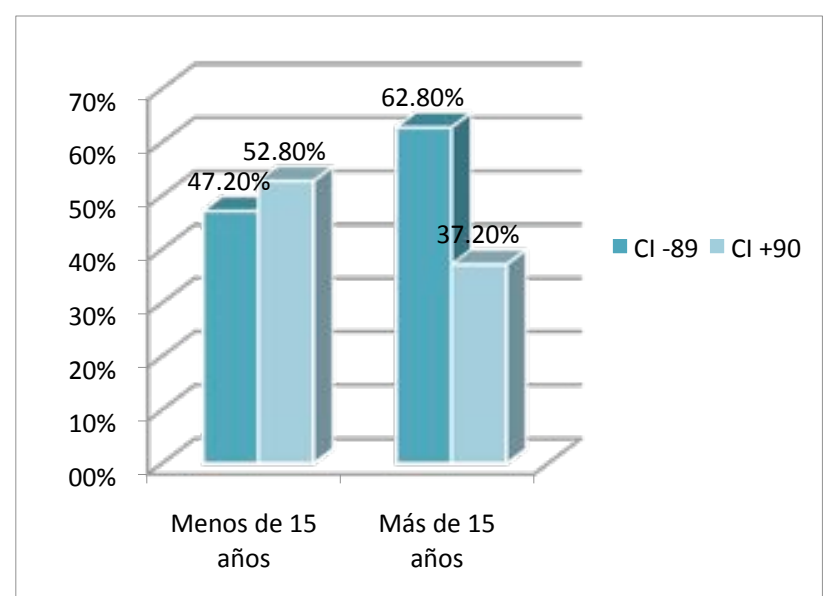

Gráfico 4. Relación del coeficiente de inteligencia del WAIS con el mayor o menor tiempo de evolución de las crisis. ${ }^{*} \mathrm{Chi} 2 \mathrm{p}=0.386$.

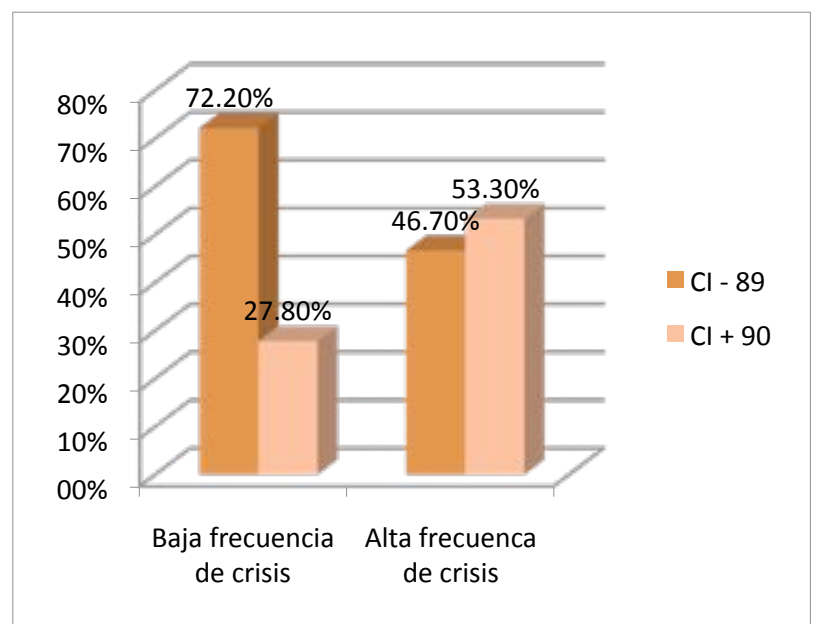

Gráfico 5. Relación de la frecuencia de las crisis con el $\mathrm{Cl}$ del WAIS. ${ }^{*}$ Chi2 $\mathrm{p}=0.0171$ y OR=2.971.

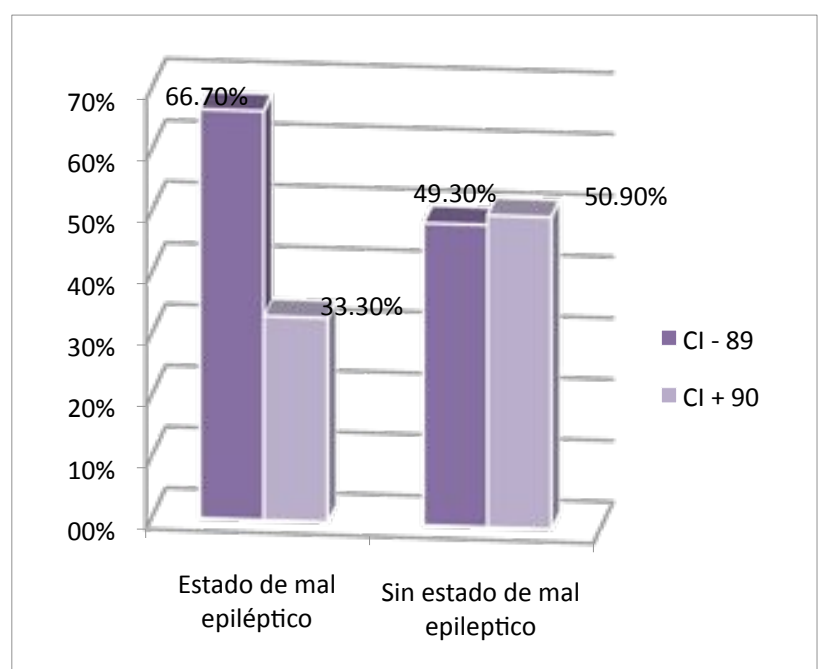

Gráfico 6. Relación del antecedente de mal epiléptico o más de 100 crisis en un mes con el nivel de $\mathrm{Cl}$. ${ }^{*} \mathrm{Chi} 2 \mathrm{p}=0.0351$ y OR 2.058 considerado significativo.
Índice de deterioro: De 200 pacientes estudiados, 106 (53\%) tenían menos de 15 años de evolución de las crisis; de estos, $46(43,4 \%)$ no tenían deterioro y $60(56,6 \%)$ sí lo tenían. Tenían 15 y más años de evolución de las crisis 94 pacientes $(47 \%)$, de los cuales $54(57,4 \%)$ estaban sin deterioro y 40 $(42,6 \%)$ con deterioro. La comparación mediante Chi2 fue de $p=0.655, O R=0.5679$, considerada no significativa.

\section{Elevada frecuencia de crisis desde el inicio de la enfermedad}

Coeficiente de inteligencia (CI). De los 200 pacientes estudiados, $54(27 \%)$ habían mantenido una frecuencia de crisis de más de una crisis/mes desde el inicio de su enfermedad, la que era considerada elevada; de estos, $39(72,2 \%)$ presentaban un $\mathrm{Cl}$ por debajo de 89 , y $15(27,8 \%)$ por encima de 90 . También, 45 $(22,5 \%)$ habían mantenido desde el inicio una frecuencia de al menos una crisis anual; de estos, $21(46,7 \%)$ tenían un $\mathrm{Cl}$ por debajo de 89 , y $24(53,3 \%)$ por encima de 90 . La comparación mediante Chi2 $\mathrm{p}=0.0171$ y $\mathrm{OR}=2.971$, considerada como significativa (ver gráfico 5 ).

Índice de deterioro. De los 200 pacientes, 54 (27\%) presentaban más de una crisis por mes desde el inicio de su enfermedad; de estos, 27 (50\%) tenían un $\mathrm{Cl}$ por debajo de 89 y un cifra similar por encima de 90 . También 45 pacientes no tenían apenas crisis (una o menos crisis en un año), de los cuales $24(53,3 \%)$ no tenían deterioro y $21(46,7 \%)$ sí lo tenían.

\section{Estado de mal epiléptico o más de 100 crisis en un año}

Coeficiente de inteligencia ( $\mathrm{Cl})$. Del total de 200 pacientes con epilepsia estudiados, 60 (30\%) referían antecedentes de más de 100 crisis o estados de mal epiléptico; de estos, 40 (66,7\%) presentaban $\mathrm{Cl}$ por debajo de 89 y 20 (33,3\%) por encima de 90. De los 140 (70\%) sin antecedentes de estado de mal epiléptico o más de 100 crisis en un año, 69 (49,3\%) tenían $\mathrm{Cl}$ por debajo de 89 y $71(50,7 \%)$ por encima de 90 . La comparación entre grupos mostró un Chi2 $\mathrm{p}=0.0351$ y OR 2.058, considerado significativo (ver gráfico 6).

Índice de deterioro. Del total de 200 pacientes, 60 (30\%) referían antecedentes de estado de mal epiléptico o más de 100 crisis en un año; de estos, 24 (40\%) no tenían deterioro y 36 (60\%) sí lo tenían. Mientras que de los 140 (70\%) que no refirieron antecedentes de más de 100 crisis de epilepsia en un año o estado de mal epiléptico, 76 (54,3\%) estaban sin deterioro y $64(45,7 \%)$ con deterioro. La comparación entre estos grupos mostró $p=0.0896$ y OR 0.5614 , considerada no significativa.

\section{Tipos de crisis de epilepsia}

Coeficiente de inteligencia ( $\mathrm{Cl}$ ). De los 200 pacientes estudiados, $19(9,5 \%)$ tienen solamente crisis generalizadas; de estos, 10 $(52,6 \%)$ tienen un $\mathrm{Cl}$ por debajo de 89 y $9(47,4 \%)$ uno por encima de 90 . Además, 38 (19\%) tienen solo crisis focales; de estos, $18(47,4 \%)$ con un $\mathrm{Cl}$ menor de 89 y 20 (52,6\%) con uno mayor de 90 . Por último, el grupo de los que tenían crisis focales secundariamente generalizadas alcanzaba los 143 pacientes $(71,5 \%)$, de los cueles 81 (56,6\%) tenían $\mathrm{Cl}$ menor de 89 y 62 $(43,4 \%)$ de más de 90 . Las comparaciones se pueden observar en el gráfico 7. 


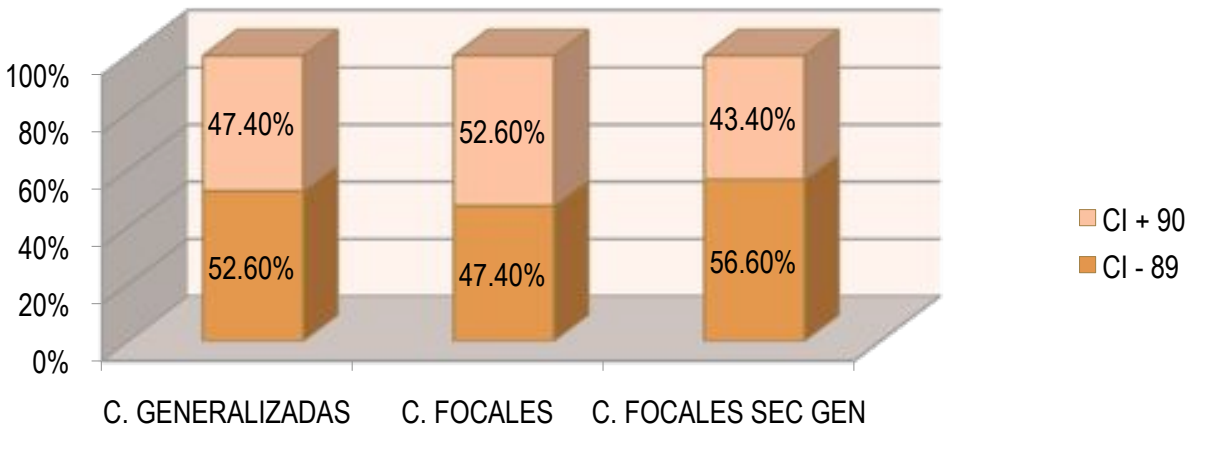

Gráfico 7. Relación del tipo de crisis de epilepsia con el coeficiente de inteligencia en 200 pacientes con epilepsia. *ANOVA p=0.0078

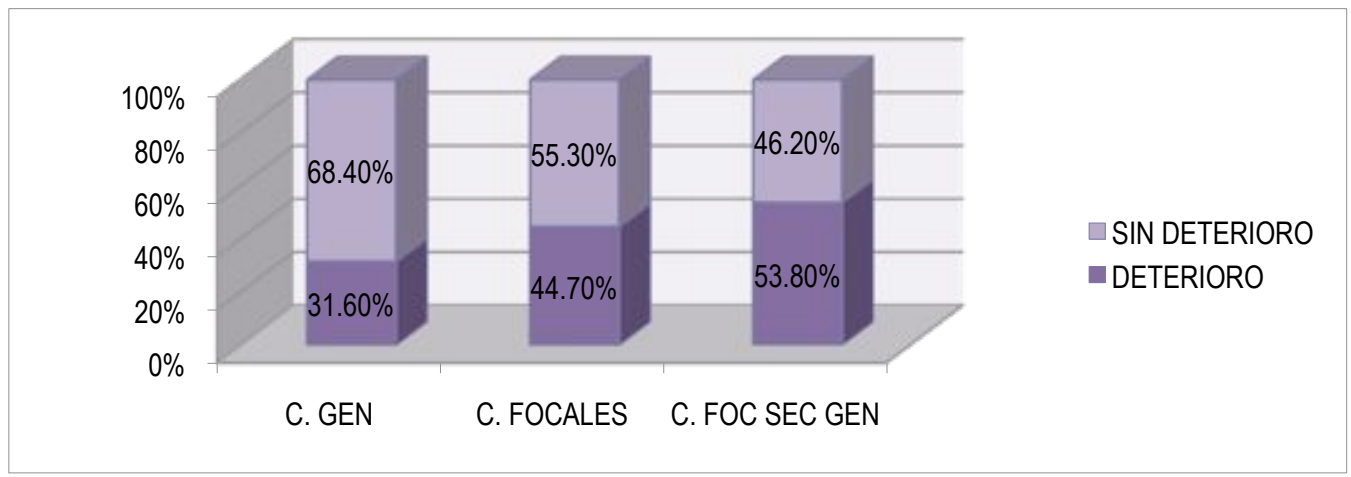

Gráfico 8. Relación del tipo de crisis de epilepsia con el deterioro en 200 pacientes con epilepsia. *ANOVA p=0.0029

En primer lugar, las crisis que más afectan el $\mathrm{Cl}$ son las crisis focales secundariamente generalizadas.

Índice de deterioro. De 200 pacientes estudiados, 19 $(9,5 \%)$ sufrían de crisis generalizadas; de estos, $6(31,6 \%)$ tenían deterioro y $13(68,4 \%)$ no lo tenían. Además, 38 (19\%) tenían crisis focales; de esos, 17 (44,7\%) tenían deterioro y 21 (55,3\%) no lo tenían. Por último, el grupo mayor de 143 pacientes $(71,5 \%)$ tenía deterioro y 66 pacientes $(46,2 \%)$ no lo tenían. Las crisis focales secundariamente generalizadas son las que más se relacionan con el deterioro (ver gráfico 8 ).

\section{Síndromes epilépticos}

En 200 pacientes con epilepsia, había 119 (59,5\%) con síndromes epilépticos focales del lóbulo temporal, mientras que 73 $(36,6)$ sufrían síndromes epilépticos focales del lóbulo frontal. Coeficiente de inteligencia. De los 119 (59,5\%) pacientes con síndromes epilépticos focales del lóbulo temporal, 67 (56,3\%) tenían un $\mathrm{Cl}$ por debajo de 89 , mientras que $52(43,7 \%)$ tenían uno por encima de 90 . De los $73(36,5 \%)$ pacientes con síndromes epilépticos focales del lóbulo frontal, 37 (50,7\%) tenían Cl por debajo de 89 , mientras que 36 (49.3\%) tenían uno por encima de 90. La comparación entre ambos grupos mediante Chi2 mostró que tiene una $\mathrm{p}=0.5424$ y un $\mathrm{OR}=1.254$, considerada no significativa. Por lo que ninguna de los dos síndromes epilépticos focales conducía a disminución del $\mathrm{Cl}$.

Índice de deterioro. Del los 119 (59,5\%) pacientes con síndromes epilépticos focales del lóbulo temporal encontramos que
$58(48,7 \%)$ no tienen deterioro y $61(51,3 \%)$ sí lo tienen; mientras que de los 73 pacientes con síndrome epilépticos focales del lóbulo frontal, $36(49,3 \%)$ no tienen síndrome de deterioro y $37(50,7 \%)$ sí lo tienen. La comparación entre ambos grupos mediante Chi2 mostró una $\mathrm{p}=0.9383$ y un $\mathrm{OR}=0.9772$, considerados no significativos. Por lo que ninguno de los dos síndromes epilépticos focales conducía a presentar un índice de deterioro positivo.

\section{Discusión}

No es raro que, al estudiar grupos de pacientes con epilepsia, se encuentren en ellos niveles exaltados de déficit de inteligencia. En este sentido, se ha visto que la prevalencia de epilepsia es substancialmente mayor en personas con inestabilidad de la inteligencia que en la población supuestamente sana (Martin \& Brown, 2009).

Los resultados de este estudio de población adulta con epilepsia, en el que se excluyó a quienes sufrían de encefalopatía epiléptica, manifestaron en un 33\% una inteli-gencia deficitaria, a lo que se añade que el $50 \%$ presentaba elementos de deterioro de la inteligencia. Es evidente que la merma en la inteligencia puede llevar a un detrimento en la calidad de vida de estos pacientes, lo que preocupa al atender pacientes adultos con este mal (Jacoby \& Baker, 2008).

Según una de las leyes de la epidemiología, las enfermedades no se asocian por simple azar, sino por un conjunto de factores de riesgo que pueden provocar esta unión. Los principales factores de riesgo presentes en el paciente con epilepsia y 
que pueden inducir trastornos cognitivos son divididos en biológicos, psicosociales y medicamentosos, para ser estudiados en un conjunto de pacientes con epilepsia (Hermann \& Whitman, 1991). Para autores que han tratado el tema de la inteligencia en pacientes adultos con epilepsia, estos factores pueden ser definidos de forma más directa como: a) factores directamente relacionados con las crisis o el tipo de epilepsia, entre los que se incluyen los llamados factores biológicos o neurobiológicos; b) factores relacionados con el tratamiento médico, como los fármacos antiepilépticos el tratamiento quirúrgico o cambios en el estilo de vida del paciente; c) estados psicopatológicos y conductuales asociados, y aspectos medio ambientales, como los estigmas, el soporte económico, oportunidades vocacionales, y d) el grado de adaptación de las personas a la epilepsia (Martin \& Brown, 2009).

En este trabajo nos hemos concentrado solo en los factores biológicos de riesgo. Para muchos son más los más importantes (Engel \& Pedely, 1997) y también los más discutidos (Jokeit \& Schacher, 2004), por lo que consideramos importante resaltar cómo se comportan los mismos en una población adulta con epilepsia, lo que puede permitir al médico de asistencia augurar el deterioro de la inteligencia asociado a estos enfermos y, lo más importante, evitar en algunos casos que esto se produzca.

De esta forma, podemos señalar que, en los pacientes estudiados, las noxas cerebrales, tales como las distocias del parto, los traumas craneoencefálicos, las encefalitis y el alcoholismo crónico - causas frecuentes de lesiones cerebrales-, no fueron relacionadas, según nuestros resultados, con un $\mathrm{Cl}$ bajo o un índice de deterioro positivo, como se ha visto en otros estudios (González-Pal, et al., 2009; Quintana-Mendoza, et al., 2004).

Con base en lo observado, los enfermos que habían iniciado las crisis de epilepsia por debajo de los 15 años de edad tenían con mayor frecuencia $\mathrm{Cl}$ por debajo de 89, distinto de quienes comenzaban sus crisis por encima de esta edad, lo que demuestra que por debajo de los 15 años las crisis de epilepsia pueden detener el desarrollo de la inteligencia con afectación del $\mathrm{Cl}$, como lo corroboran algunos estudios (Blumer \& Hermann, 1993; Campos-Castello, 2006).

Los pacientes con evolución prolongada de las crisis de epilepsia, llevadas a un límite de más de 15 años de episodios ictales, presentaron con más frecuencia un $\mathrm{Cl}$ por debajo de 89 , lo que significa que a mayor tiempo de evolución de las crisis existe mayor deterioro. Aunque algunos artículos coinciden en este aspecto, no dejan de reconocer que a este tiempo mayor de evolución de crisis se añade el impacto negativo de las crisis según su tipo (Dikmen \& Matthews, 1977; GonzálezPal, et al., 2009; Jokeit \& Ebner, 1999; Stefan \& Pauli, 2002).

En el grupo de enfermos con elevada frecuencia desde el inicio de la enfermedad, calculado con una media de más de tres crisis en un mes, se observa con más frecuencia un $\mathrm{Cl}$ menor de 89 , y la probabilidad de los pacientes con elevado número de crisis de epilepsia de tener un $\mathrm{Cl}$ bajo es tres veces mayor. En este aspecto, existe coincidencia con este resultado (González-Pal, et al., 2009). También se observó que los ante-cedentes de estados de mal epiléptico o más de 100 crisis en un año inducían al déficit de $\mathrm{Cl}$. En cuanto al estado de mal epiléptico y el deterioro a largo plazo, hay algunos trabajos que los apoyan (Holmes, Dodrill, Wilkus et al., 1998; Thompson \& Duncan,
2005). Adicionalmente, una revisión de nueve estudios longitudinales, que miden el funcionamiento intelectual de niños con pobre control de las crisis de epilepsia, encuentra en todos los estudios una disminución del coeficiente de inteligencia (Dodrill, 2004). Hay más consenso en considerar que más que la frecuencia de los episodios ictales es más decisiva la severidad de éstos (Dodrill, 1986; Dodrill \& Troupin, 1976; Jokeit \& Ebner, 2002; Oyegbile, Dow, Jones et al., 2004). Sin embargo, hay quienes no descubren vínculo entre el deterioro y la frecuencia de las crisis (Holmes, et al., 1998).

Una de las variables más discutidas es la de los tipos de crisis de epilepsia y su deterioro. Según autores como Hoffmann, Zhao y Holmes (2004), las crisis generalizadas (tónicoclónicas, tónicas o clónicas) se relacionan con mayor deterioro por los múltiples traumas que sufren estos pacientes. También, hay artículos que consideran que en los pacientes con epilepsias parciales del lóbulo temporal hay mayor tendencia al deterioro cognitivo (Thompson \& Duncan, 2005). Nuestros resultados apoyan ambos planteamientos, pues los pacientes con $\mathrm{Cl}$ más bajo y mayor deterioro tenían crisis parciales secundariamente generalizadas (más del $80 \%$ con crisis parciales complejas características del lóbulo temporal), seguidos de los que solo tenían crisis generalizadas.

En nuestro estudio con pacientes adultos no se muestran diferencias entre los pacientes con epilepsia focales temporales y frontales, lo que es un hallazgo frecuente en estudios con niños, en quienes se observa que el funcionamiento cognitivo de la epilepsia es mejor en las epilepsias temporales que en las frontales, en las que, a su vez, son mejores que en las epilepsia generalizadas sintomáticas (Nolan, et al., 2003).

Finalmente, podemos decir que se considera elevada la merma de la inteligencia en los pacientes adultos con epilepsia, la que, según nuestros resultados, está muy relacionada con un conjunto de factores biológicos en los pacientes, entre los que se destacan:

- Pacientes que comenzaban las crisis de epilepsia por debajo de los 15 años de edad.

- Aquellos con cronicidad de sus crisis de epilepsia, con más de 15 años de evolución de estas.

- Los que desde el inicio de la enfermedad mantienen una frecuencia de crisis superior a una por mes y enfermos con antecedentes de haber tenido más de 100 crisis en un año de su vida.

- Los que sufren de crisis generalizadas o crisis parciales secundariamente generalizadas.

El conocimiento y la detección de estos factores en etapas tempranas de la vida puede dar un pronóstico adecuado a largo plazo de la inteligencia del paciente $y$, además, ese déficit puede se rectificado si se aprovecha el proceso de neuroplasticidad y se ejercita más a nuestro niños y jóvenes con epilepsia en funciones que pueden estar alteradas. Hay que tener presente que los pacientes con epilepsia y merma de funciones cognitivas de la inteligencia tienen una peor calidad de vida, lo que requiere esforzarse, primero, en detectar cuales son los factores que más influyen y, posteriormente, en trazar una estrategia que sirva para minimizar estos trastornos. 


\section{Referencias}

Acevedo, C., Campos, M., Mesa, T. \& Nuñez, L. (Eds.). (2006). Epilepsias: lo que usted debe saber sin ser médico. Santiago de Chile: BHA Impresores SA.

Beghi, E. (2009). The concep of the epilepsy Syndrome: How useful is it in clinical practice? Epilepsia, 50(Suppl 5), 4-10.

Binnie, C., Debets, R., Engelsman, M. et al. (1989). Double-blind crossover trial of lamotrigine (Lamictal) as add-on therapy in intractable epilepsy. Epilepsy Res, 4, 222-229.

Blumer, D. \& Hermann, B. (1993). Behavioral and emotional adjustment in epilepsy. Issues in epilepsy and quality of life. Landover: Epilepsy. Foundation of America, 1-8.

Brown, S. (2006). Deterioration. Epilepsia, 47(Suppl 2), 19-23.

Campos-Castello, J. (2006). Neuropsicología de la epilepsia: ¿Qué factores están implicados? Rev Neurol, 43(Suppl 1), S59-S70.

Caramelli, P. \& P, M.C. (2005). Dementia Associated with Epilepsy. International Psychogeriatrics, 17(suppl1), 195-206.

Dikmen, S. \& Matthews, C. (1977). Effect of major motor seizure frequency upon cognitive-intellectual functions in adults. Epilepsia, 18, 21-29.

Dodrill, C. (1986). Correlates of generalized tonic-clonic seizures with intellectual, neuropsychological, emotional, and social function in patients with epilepsy. Epilepsia, 27, 399-341.

Dodrill, C. (2004). Neuropsychological effects of seizures. Epilepsy Behav, 5(suppl 1), S21-24.

Dodrill, C. \& Troupin, A. (1976). Seizures and adaptative abilities: a case of identical twins. Archives of Neurology, 33, 604-607.

Engel, J. (2006). A proposed diagnostic scheme for people with epileptic seizures an with epilepsy:report of the ILAE task force on classification and terminology. Epilepsia, 42, 796-803.

Engel, J. \& Pedely, J. (Eds.). (1997). Neuropsychological evaluation. (Lippincott-Raven ed. Vol. 1). Philadelphia.

González-Pal, S., Quintana-Mendoza, J., Fabelo-Roche, J., Delgado, E.G. \& Moré-Iglesias, S. (2009). Principales factores multicausales en la epilepsia que inducen al deterioro de funciones cognitivas. Revista Ecuatoriana de Neurología, 18(1-2), 31-32.

Hermann, B., Sidenberg, M. \& Bell, B. (2000). Psychiatric comorbility in chronic epilepsy: identification, consequences, and treatment of mayor depression. Epilepsia, 41(2), 31-41.

Hermann, B. \& Whitman, S. (1984). Behavioral and personality correlates of epilepsy: a review, methodological critique, and conceptual model. Psychological Bulletin, 95(3), 451-497.

Hermann, B.P. \& Whitman, S. (1991). Neurobiológical, psychosocial and pharmacological factors underlying interictal psychopatology in epilepsy. In S. D, T. D \& T. M (Eds.), Advances in Neurology. Neurobiológical, psychosocial and pharmacological factors underlying interictal psychopatology in epilepsy, pp. 439-452.

Hoffmann, A., Zhao, Q. \& Holmes, G. (2004). Cognitive mpairment followingstatus epilepticus and recurrent seizures during early development: support for the "two-hit hypothesis". Epilepsy \& Behavior, 5, 873-877.

Holmes, M., Dodrill, C., Wilkus, R. et, al. (1998). Is partial epilepsy progressive? Ten-year folloup of EEG and neuropsychological changes in adults with partial seizures. Epilepsia, 39, 1189-1193.

Jacoby, A. \& Baker, G. (2008). Quality-of-life trajectories in epilepsy: a review of the literature Epilepsy \& Behavior, 12, 557-571.

Jokeit, H. \& Ebner, A.A. (2002). Effects of chronic epilepsy on intellectual functions. Progress in Brain Research, 135, 455-463.

Jokeit, H. \& Schacher, M. (2004). Neuropsychological aspects of type of epilepsy and etiological factors in adults. Epilepsy \& Behavior, 5, S14-S20.

Jokeit, J.H. \& Ebner, A. (1999). Long term effects of refractory temporal lobe epilepsy on cognitive abilities: a cross sectional study. Journal of Neurology, Neurosurgery \& Psychiatry, 67, 44-50.

Martin, J.P. \& Brown, S.W. (2009). Best clinical and research practice in adults with an intellectual disability. Epilepsy \& Behavior, 15, S64S68.
Nolan, M. A., Redoblado, M.A., Lahc, S., Sabaz, M., Lawson, J.A., Cunningham, A.M., et al. (2003). Intelligence in childhood epilepsy syndromes. Epilepsy Research, 53, 139-150.

Oyegbile, T., Dow, C., Jones, J. et al. (2004). The nature and course of neuropsychological morbidy in chronic temporal lobe epilepsy. Neurology, 62, 1736-1742.

Quintana-Mendoza, J., González-Pal, S. \& González-Rodríguez, C. (2004). Wais como instrumento evaluador del deterioro cognitivo en las psicosis epilepticas. Psiquiatria.com, 8(3). Retrieved from http://www.psiquiatria.com/psiquiatria/revista/131/16079/?++inte ractivo

Stefan, H. \& Pauli, E. (2002). Progressive cognitive decline in epilepsy: an indication of ongoing plasticity. Progress in Brain Research, 135, 409-417.

Thompson, P. \& Duncan, J. (2005). Cognitive Decline in Severe Intractable Epilepsy. Epilepsia, 46, 1780-1787.

Ure, J. (2004). Deterioro cognitivo en pacientes epilépticos. Revista Argentina de Neuropsicología, 2, 1-14.

Wechsler, D. (Ed.). (1955). La medición de la Inteligencia en Adultos. La Habana, Cuba. 